\title{
Therapeutic approach of human peritoneal carcinomatosis with Dbait in combination with capnoperitoneum: proof of concept
}

\author{
Wiebke Solass $\cdot$ Aurélie Herbette $\cdot$ Tina Schwarz \\ Alexander Hetzel • Jian-Sheng Sun • \\ Marie Dutreix $\cdot$ Marc A. Reymond
}

Received: 2 June 2011/Accepted: 19 September 2011/Published online: 1 November 2011

(c) The Author(s) 2011. This article is published with open access at Springerlink.com

\begin{abstract}
Background Peritoneal carcinomatosis is an unmet medical need. Laparoscopy offers a unique opportunity to control and to steer the operating environment during surgery by loading carbon dioxide with a therapeutic substance and creating the so-called therapeutic capnoperitoneum. We have treated a human sample of peritoneal carcinomatosis from an endometrial adenocarcinoma ex vivo just after surgery.

Methods A nontoxic therapeutic agent (Dbait) was aerosolized into a box containing diseased human peritoneum
\end{abstract}

Presented at the Annual Congress of the European Association for Endoscopic Surgery (EAES), Torino, Italy, June 2011.

W. Solass · M. A. Reymond

Department of Surgery, Otto-von-Guericke University,

Magdeburg, Germany

A. Herbette $\cdot$ M. Dutreix

UMR ETIC, Institut Curie, Orsay, France

A. Herbette $\cdot$ J.-S. Sun $\cdot$ M. Dutreix

DNA Therapeutics, Evry, France

T. Schwarz · A. Hetzel

Reger Medizintechnik, Rottweil, Germany

J.-S. Sun

USM503 Muséum National d'Histoire Naturelle, Paris, France

M. A. Reymond ( $\square)$

Department of Surgery, Protestant Hospital, Bielefeld, Germany

e-mail: marc.reymond@evkb.de

M. A. Reymond

Klinik für Allgemein-und Viszeralchirurgie, Evangelisches Krankenhaus Bielefeld, Schildescher Str 99, 33611 Bielefeld, Germany under a pressure of $12 \mathrm{mmHg} \mathrm{CO}$. Dbait (noncoding DNA fragments) acts through jamming DNA damage sensing and signaling, ultimately inhibiting DNA repair system of cancer cells. Dbait were coupled to cholesterol molecules to facilitate intracellular uptake, and to Cyanine (Cy5) to allow detection by fluorescence. In a control experiment, the same solution was applied to the other half of the sample using conventional lavage.

Results Physical results revealed fluorescence within the tumor up to $1 \mathrm{~mm}$ depth in the therapeutic capnoperitoneum sample and no uptake in the lavage sample. Biological results showed intranuclear phosphorylation of $\mathrm{H} 2 \mathrm{AX}$ in the nebulized sample and no activity in the lavage sample. Importantly, tumor nodules showed more activity than the neighbor, normal peritoneum. Detection of histone gamma-H2AX (phosphorylated H2AX) reveals activation of DNA-dependent protein kinase (DNA-PK) by Dbait, which has been shown to be the key step for sensitization to genotoxic therapy.

Conclusions Dbait are taken up by cancer cells and have a biological activity up to $1 \mathrm{~mm}$ depth. Nebulization of the molecule is significantly more effective than conventional lavage. This proof of principle supports the need for clinical studies applying therapeutic capnoperitoneum together with Dbait for treating peritoneal carcinomatosis.

Keywords Laparoscopic surgery - Peritoneum-therapy · Nebulizer · Cancer · Pneumoperitoneum · Dbait

Peritoneal carcinomatosis (PC) is a devastating form of cancer progression with a very poor prognosis. Chemotherapy has only limited effect due to drug resistance in advanced tumor stages and to poor bioavailability within the abdominal cavity after systemic drug application. Current therapy, 
when possible, includes cytoreductive surgery associated or not with hyperthermic intraperitoneal chemotherapy [1]. However, this therapy is linked to significant morbidity and mortality, and results are still limited [2]. Thus, there is a need for novel therapeutic approaches, including preventive strategies during initial surgery [3].

The ability of cancer cells to recognize damage and initiate DNA repair is an important mechanism of resistance to treatment. Pharmacological inhibition of DNA repair for a short period during treatment has the potential to make cancer cells more vulnerable to the damaging effects of therapy, therefore increasing the response to treatment [4]. Dbait acts by baiting and hijacking the enzyme complexes that repair DNA double strand breaks (DSB), diverting them away from their primary objective, the DSBs on chromosomes [5]. Such DSB are currently caused by treatment, such as radiotherapy (RT) and DNAdamaging chemotherapy (CT). Dbait increases the sensitivity of HNCC, melanoma and glioblastoma to irradiation [6], and the sensitivity of colorectal cancer to chemotherapy [7]. However, a limitation of this approach is tumor access and penetration into tumor nodes, so that it can only be applied to superficially spreading tumors.

Laparoscopy offers a unique opportunity to control and to steer the operating environment during surgery, by loading carbon dioxide with a therapeutic substance and creating the so-called therapeutic capnoperitoneum [8]. Recently, we have shown that therapeutic peritoneum allows homogeneous distribution of a therapeutic substance within the peritoneal cavity and an improved penetration depth into normal and diseased tissue compared with conventional peritoneal lavage [9].

However, working with toxic therapeutic aerosols is only possible under strongly regulated conditions. Interestingly, preclinical studies with Dbait in the primate have shown no toxicity of the molecule on healthy tissues (data on file). Thus, there is a rationale for combining Dbait with therapeutic capnoperitoneum to obtain a synergy. In particular, local increase in chemosensitivity could improve efficacy of both intraperitoneal and systemic chemotherapy on PC.

The general purpose of the present ex vivo experiment was to test possible advantages of combining both technologies for treating a human PC tumor nodule, in particular concerning tumor access (distribution and penetration). A second specific goal was to examine whether genotoxicity can be demonstrated in a tumor nodule of human PC after loading capnoperitoneum with Dbait.

\section{Material and methods}

The current study was designed to investigate the biodistribution of Dbait in a PC nodule ex vivo, immediately after surgical resection. For this purpose, a tumor nodule was exposed to Dbait aerosol using the micropump (MIP) spraying device under $12 \mathrm{mmHg}$ of $\mathrm{CO}_{2}$ pressure in a closed box modeling the peritoneal cavity. An electrical gradient was generated to focus drug distribution onto the peritoneal surface and to enhance tissue penetration (Fig. 1). As controls, two further experiments were conducted: (1) the experiment as above without Dbait, and (2) lavage of a PC nodule with Dbait in a glucose solution.

The following parameters have been measured:

(a) Surface homogeneity of Dbait distribution within the simulated peritoneal cavity,

(b) Dbait diffusion into the peritoneal nodule, and

(c) Dbait's bioactivity in cancer cells under oxidative stress.

Study design

This ex vivo study involved tumor tissue from a single human patient suffering PC from endometrial origin. Because patient therapy was not influenced by the experiment, no authorization from an ethical committee was required. Ex vivo research on human tissue is allowed at our institution by a valid authorization of the common ethics committee of the Westfalian University-Medical Chamber of Westfalia-Lippe (AeKWL).

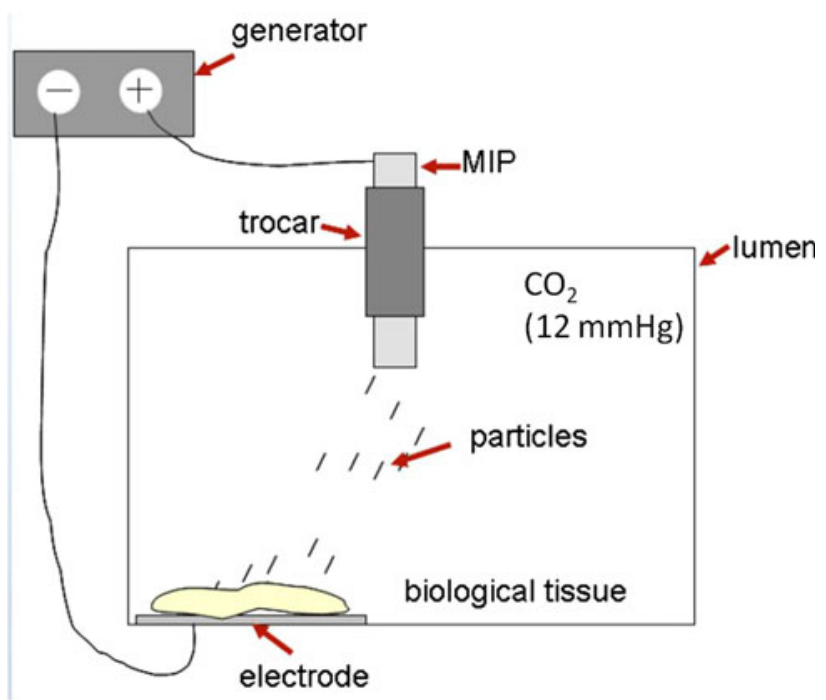

Fig. 1 Laparoscopy-like ex vivo experiment on fresh operation specimen of diseased human peritoneum (peritoneal carcinomatosis from endometrial origin). Therapeutic capnoperitoneum $(12 \mathrm{mmHg})$ was established in a plastic box. An electrostatic gradient of $60 \mathrm{~V}$ was generated between the MIP and the biological tissue, placed on a neutral electrode. MIP micropump 
Nebulizer

The nebulizer has been described elsewhere [9] and consists of several components, including an injector, a line, and a nozzle. The nozzle had a diameter of $0.2 \mathrm{~mm}$, and a pressure of approximately 8 bars was delivered upfront of the nozzle. The nebulizer was inserted through a 10-mm trocar and generated an aerosol within the plastic box mimicking the abdominal cavity. The gaseous phase consisted of $\mathrm{CO}_{2}$, the liquid phase of microparticles of Dbait (100 $\mu \mathrm{g} / \mathrm{ml})$.

Patient characteristics, cytoreduction, and intraperitoneal hyperthermic chemotherapy

A female patient suffering PC from endometrial cancer was selected for the experiment and gave her written, informed consent. Chloroquine $100 \mathrm{mg}$ qd was administered for 1 week before the operation. First, a complete cytoreduction was achieved (CRS). In a second step, intraperitoneal hyperthermic chemotherapy was applied with cisplatin $75 \mathrm{mg} / \mathrm{m}^{2}$ body surface and doxorubicin $15 \mathrm{mg} / \mathrm{m}^{2}$ body surface, according to usual protocols.

\section{Tumor characteristics}

Immediately after surgical cytoreduction, a peritoneal fragment of approximately $10 \times 10 \mathrm{~cm}$, showing macroscopic peritoneal nodules, was selected for experimental purpose. The remaining surgical specimen was sent to the pathologist for diagnostic purpose. Microscopic analysis showed a poorly differentiated (G3) clear cell adenocarcinoma with few small necrosis foci.

\section{Experimental protocol}

The three surgical samples were treated as follows:

a) The first peritoneal nodule was inserted into the experimental box, placed on the electrode, and exposed to $\mathrm{CO}_{2}$ capnoperitoneum at room temperature for 30 minutes. After dilution into $90 \mathrm{ml}$ of glucose $5 \%, 9 \mathrm{mg}$ of Cy5-labelled Dbait (1:1 dilution with non fluorescent Dbait) was aerosolized into the box.

b) A second peritoneal nodule was exposed to "peritoneal lavage" using $0.1 \mathrm{mg} / \mathrm{ml}$ Dbait-Cy5 diluted into $10 \mathrm{ml}$ of glucose $5 \%$.

c) A third peritoneal nodule was exposed to identical conditions as (a) without Dbait (negative control).

Immediately after the experiment, all three samples were split into two halves: the first half was fixed in formalin solution and sent to the pathologist, the second half was immediately frozen at $-80^{\circ} \mathrm{C}$ for further biological analysis.

Microscopic analysis

For analysis, formalin-fixed specimens were stained with H\&E according to usual protocols. For microscopic fluorescence analysis, sampled were fixed with PFA $4 \%$ for $20 \mathrm{~min}$ at room temperature (RT), followed by exposure to Triton $0.5 \%$ for $10 \mathrm{~min}$ at RT. Then, samples were washed out in SVF diluted $1 / 2$ in PBS solution for $1 \mathrm{~h}$ at RT, followed by incubation with $\gamma$-H2AX mouse antibody (Millipore, Billerica, MA, USA) diluted 1/200 (SVF 1/2 in PBS) for $2 \mathrm{~h}$ at RT. After washing, samples were incubated with secondary anti-mouse FITC (Sigma-Aldrich Chimie Sarl., Lyon, France) diluted 1/100 for $45 \mathrm{~min}$ at RT. Nuclei were counterstained with Dapi $0.5 \mu \mathrm{g} / \mathrm{ml}$ for $5 \mathrm{~min}$ at room temperature. Analysis was performed with an inverted microscope.

\section{Results}

The experiment was performed according to the schedule. No technical difficulty was encountered. In particular, MIP generated the Dbait-aerosol without any difficulty. Remote control of the injection device was possible without direct intervention in the operating room. All six sides of the experimental box were stained; however, an intensity gradient was observed from the bottom to the top of the box (not shown).

Microscopic analysis revealed homogeneous peritoneal distribution of Dbait-Cy5 in the therapeutic capnoperitoneum sample (arrows), only minimal uptake in the lavage sample, and no staining (or only marginal staining) in the control sample (Fig. 2). Fluorescence was detected within the tumor up to $1 \mathrm{~mm}$ depth in the therapeutic capnoperitoneum sample but not in the lavage sample (Fig. 3). Thus, aerosolization of the molecule allowed significantly better tumor access and better bioavailability of Dbait than conventional lavage.

Biological results showed intranuclear phosphorylation of H2AX in the nebulized sample and almost no activity in the lavage sample (Fig. 4). The low activity detected in the lavage sample is due to the presence of spontaneous chromosomal damage in PC cells. Detection of histone gamma-H2AX (phosphorylated $\mathrm{H} 2 \mathrm{AX}$ ) reveals the nuclear activation of DNA-dependent protein kinase (DNA-PK) by Dbait. Dbait was taken up by cancer cells, and a biological activity was detected up to $1 \mathrm{~mm}$ depth. Importantly, tumor nodules showed more activity at the tumor invasion front (Fig. 5). 

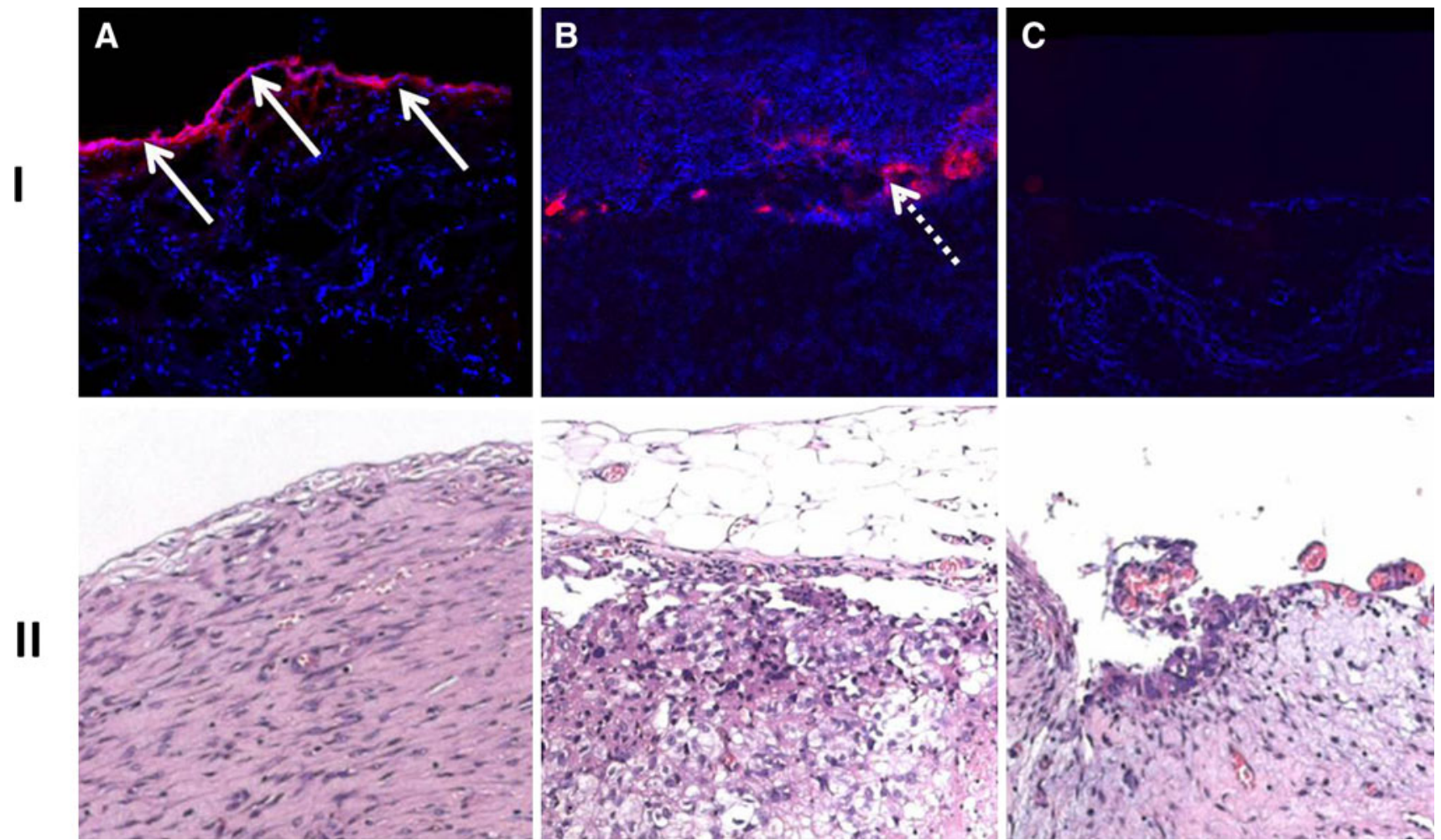

Fig. 2 Diffusion of therapeutic substance onto the peritoneum. I. Cryosection of human peritoneum, red: Dbait-Cy5 staining, blue: Dapi (nucleus). A After nebulization of Dbait, B after lavage with Dbait, C after nebulization without Dbait. Staining reveals homogeneous peritoneal fluorescence in the therapeutic capnoperitoneum sample (arrows), only minimal uptake in the lavage sample, and no staining in the control sample. II: H\&E staining
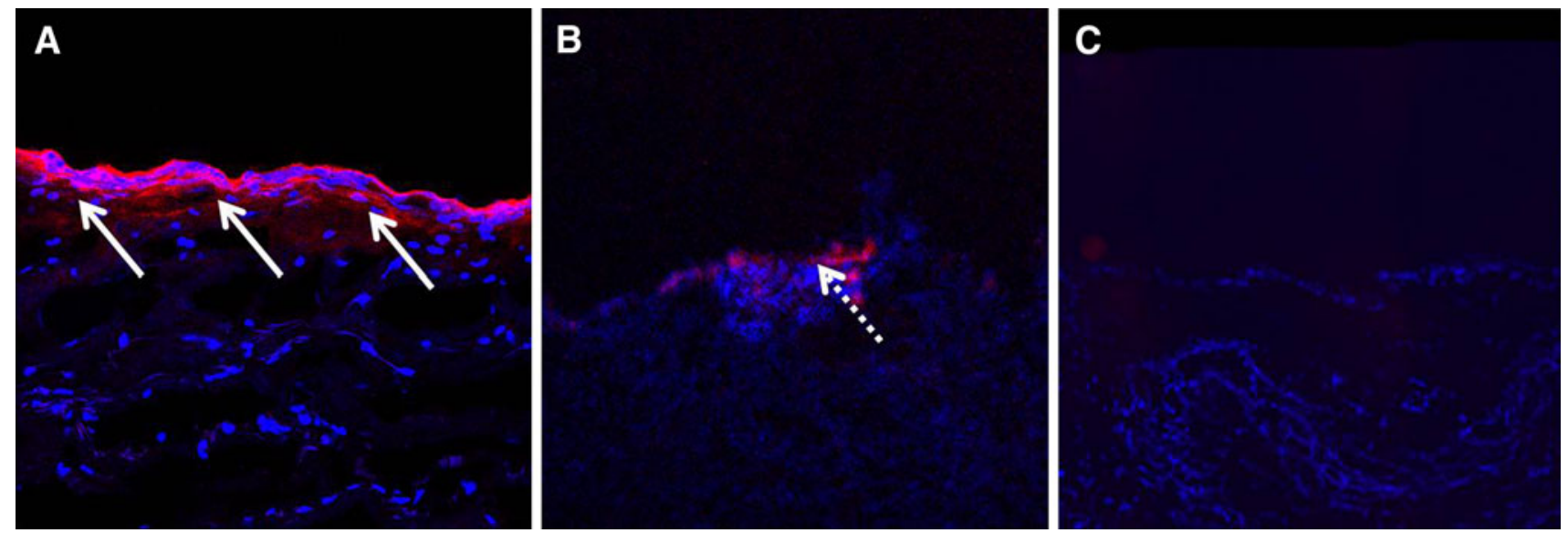

Fig. 3 Penetration of therapeutic substance into the peritoneum. Cryosection of human peritoneum, red: Dbait-Cy5 staining, blue dapi (nucleus). A After nebulization of Dbait, B after lavage with Dbait, C after nebulization without Dbait. Staining reveals fluorescence up

\section{Discussion}

This ex vivo experiment was designed to examine possible advantages of combining Dbait with therapeutic capnoperitoneum for treating a human tumor nodule of PC ex vivo, in particular concerning tumor access to seven cellular layers in the therapeutic capnoperitoneum sample (A), no tissue uptake in the lavage sample (B) and no staining in the control sample (C)

(distribution and penetration). A second specific goal was to examine whether bioactivity of Dbait could be demonstrated.

This is the first time that biological activity of Dbait is proved in human patient tissue. So far, experiments have been performed in human tumor xenograft models in the 


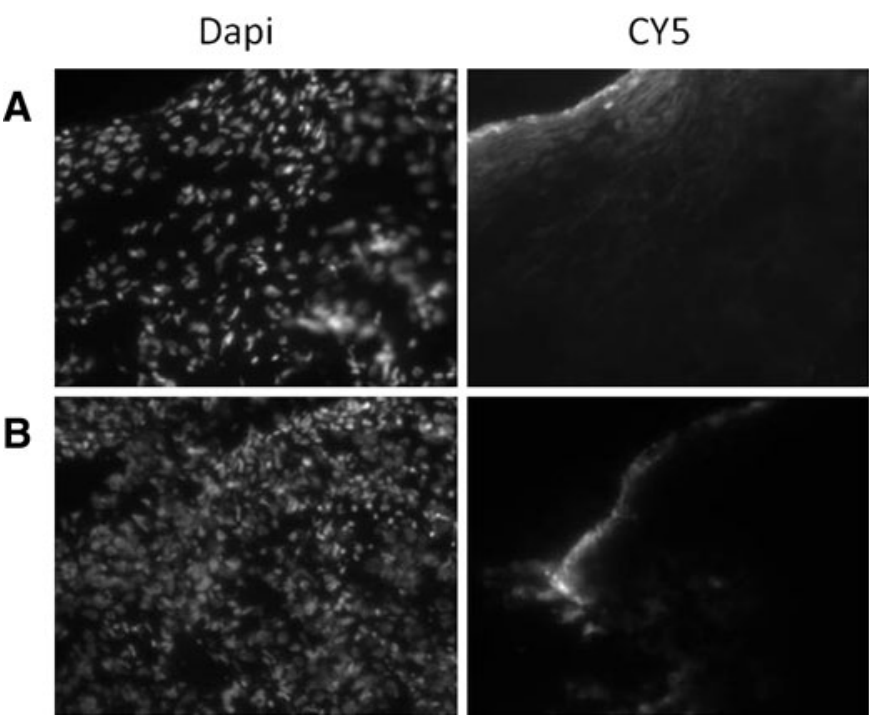

Fig. 4 Biological activity. Cryosection of human peritoneum. Detection of early Dbait activity marker: Phosphorylated H2AX. Red: Dbait-Cy5 staining, blue: Dapi staining (nucleus), green: $\gamma$-H2AX. A After nebulization of Dbait, B after lavage with Dbait. Intranuclear phosphorylation of $\mathrm{H} 2 \mathrm{AX}$ is observed the nebulized
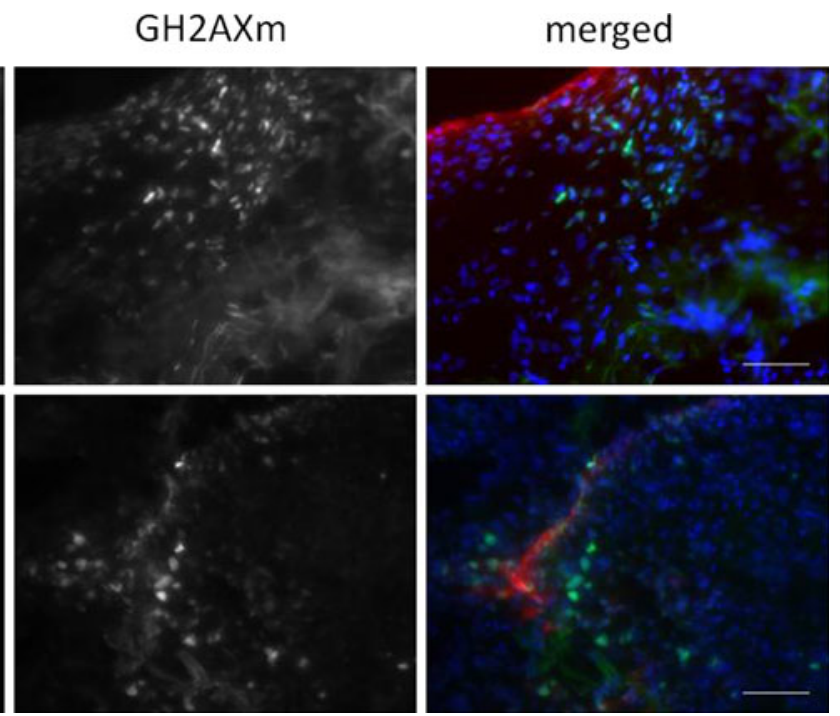

sample and only artifactual staining (no nuclear staining) in the lavage sample. Detection of histone $\gamma$-H2AX (phosphorylated $\mathrm{H} 2 \mathrm{AX}$ ) reveals activation of DNA-Phosphokinase by Dbait, which has been shown to be the key step for sensibilization to genotoxic therapy
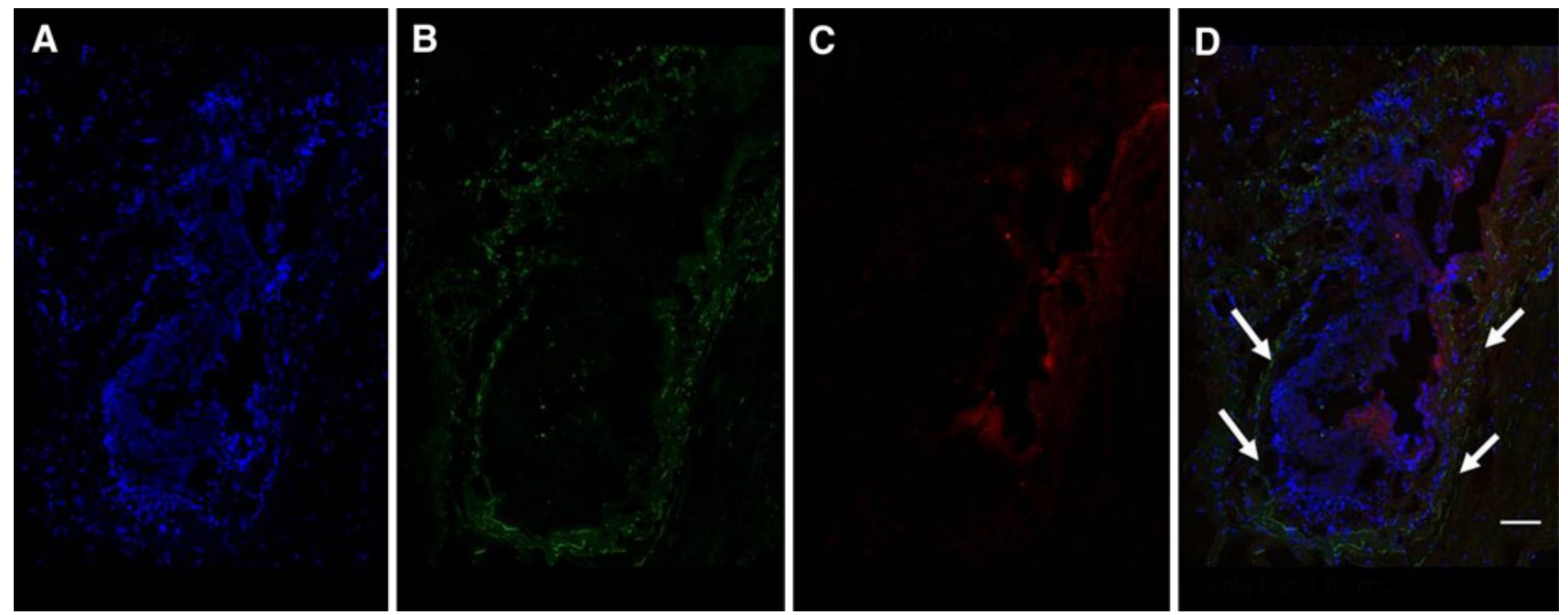

Fig. 5 Tumor specificity. Detection of early Dbait activity marker: cryosection of human peritoneum; A blue: Dapi staining (nucleus); B green: $\gamma$-H2AX after nebulization of Dbait, C red: Dbait-Cy5 staining, D merged: H2AX activation is maximal at the tumor invasion front (arrow)

animal. This is significant progress toward regulatory approval of Dbait for therapeutic use in patients.

The above-mentioned results are encouraging, because they evidence a homogeneous distribution of Dbait onto peritoneum and a better penetration into peritoneum than after conventional peritoneal lavage. This excellent bioavailability of Dbait in human peritoneal tissue ex vivo confirms the results obtained previously in the large-animal model showing a better vital staining of the abdominal cavity after aerosolization than after lavage with methylene blue [9].

Another important finding was intranuclear phosphorylation of $\mathrm{H} 2 \mathrm{AX}$ in the nebulized sample, proving Dbait uptake up to $1 \mathrm{~mm}$ depth, and showing biological activity. Detection of histone gamma-H2AX (phosphorylated $\mathrm{H} 2 \mathrm{AX}$ ) reveals nuclear activation of DNA-PK by Dbait, a significant result because this activation has been shown to be the key step for sensibilization to genotoxic therapy $[5,6]$. Moreover, this result has been obtained under oxidative stress conditions (warm ischemia) in human tumoral tissue-and not in an animal model. Importantly, tumor nodules showed more activity than the neighbor, normal peritoneum, in particular at the tumor invasion front. Thus, the present experiment delivers the proof of principle for combining Dbait with systemic or intraperitoneal 
chemotherapy to produce significant synergy. Of course, this proof of principle ex vivo should now be confirmed by corresponding in vivo studies.

Current intraperitoneal hyperthermic chemotherapy guidelines recommend the application of an intraperitoneal temperature of $41-43^{\circ} \mathrm{C}$ [10]. Recently, we observed that numerous Heat Shock proteins were phosphorylated in cells treated by Dbait. Moreover, in vitro, Dbait sensitize cells to temperature shift (unpublished data). Thus, it will be interesting to perform these efficacy studies under hyperthermic conditions to increase the biological effect of Dbait.

In this experiment, biological response was observed already after less than $1 \mathrm{~h}$. This was a surprise because preclinical data obtained in the animal model showed maximal biological response after $3 \mathrm{~h}$. This can be explained by the application of an electrostatic gradient of $60 \mathrm{~V}$, which is similar to the condition of cellular electroporation. This was obviously helpful for uptake of very large amounts of Dbait molecules. Preoperative therapy with chloroquine also might have contributed to this improved cellular drug uptake: chloroquine is known to fragilize the membranes of the endosomes, so that the release of Dbait molecules into the cytoplasm is facilitated [11].

The biological effect was achieved with a low Dbait dose, only $9 \mathrm{mg}$ for a volume corresponding to the entire abdominal cavity. Dbait effect is strictly dose-dependent, as demonstrated in several tumor systems [6]. In the preclinical model, a dose of $4 \mathrm{mg}$ was considered adequate for treating small tumor xenografts (data submitted). Thus, an excellent intracellular uptake of Dbait was achieved under experimental conditions.

Another, major point of interest is that Dbait has shown no toxicity in preclinical studies in the primate (data on file). Thus, aerosolization of Dbait might be possible in clinical practice without particular safety measures for protecting both patient and surgical team. This favorable safety profile might speed up implementation of therapeutic capnoperitoneum in cancer surgery.

In summary, combining Dbait with therapeutic capnoperitoneum appears to have several practical advantages. Peritoneal carcinomatosis is a superficial and diffuse tumor that is accessible to Dbait. Pressure enhances Dbait penetration into tumor tissue. $\mathrm{CO}_{2}$ as a carrier ensures excellent drug distribution onto the peritoneum. Application of an electrostatic gradient optimizes both drug distribution and drug uptake, probably by inducing cellular electroporation. Dbait has no toxicity demonstrated in the primate so that its clinical use appears easily possible. Finally, intraperitoneal hyperthermia might enhance the efficacy of Dbait by creating cellular stress, but this has not been investigated in the present proof-of-principle experiment.

The development of procedures easily applicable to the clinical setting that might improve outcome in PC should be given high priority. This proof-of-principle experiment opens a new avenue for treating PC that should now be followed by adequate clinical studies, a precondition for regulatory approval of this new, challenging therapeutic approach.

Disclosures J. Sun and M. Dutreix have an equity interest in DNA Therapeutics. A. Herbette is an employee of DNA Therapeutics. T. Schwarz and A. Hetzel are employees of Reger Medizintechnik. A. Hetzel has an equity interest in Reger Medizintechnik. W. Solass and M.A. Reymond have no conflicts of interest or financial ties to disclose.

Open Access This article is distributed under the terms of the Creative Commons Attribution Noncommercial License which permits any noncommercial use, distribution, and reproduction in any medium, provided the original author(s) and source are credited.

\section{References}

1. Roviello F, Caruso S, Marrelli D, Pedrazzani C, Neri A, De Stefano A, Pinto E (2011) Treatment of peritoneal carcinomatosis with cytoreductive surgery and hyperthermic intraperitoneal chemotherapy: state of the art and future developments. Surg Oncol 20(1):e38-e54

2. Baratti D, Kusamura S, Laterza B, Balestra MR, Deraco M (2010) Early and long-term postoperative management following cytoreductive surgery and hyperthermic intraperitoneal chemotherapy. World J Gastrointest Oncol 2(1):36-43

3. Kusamura S, Baratti D, Zaffaroni N, Villa R, Laterza B, Balestra MR, Deraco M (2010) Pathophysiology and biology of peritoneal carcinomatosis. World J Gastrointest Oncol 2(1):12-18

4. Helleday T, Petermann E, Lundin C, Hodgson B, Sharma RA (2008) DNA repair pathways as targets for cancer therapy. Nat Rev Cancer 8:193-204

5. Quanz M, Chassoux D, Berthault N, Agrario C, Sun JS, Dutreix M (2009) Hyperactivation of DNA-PK by double-strand break mimicking molecules disorganizes DNA damage response. PLoS One 4(7):e6298

6. Quanz M, Berthault N, Roulin C, Roy M, Herbette A, Agrario C, Alberti C, Josserand V, Coll JL, Sastre-Garau X, Cosset JM, Larue L, Sun JS, Dutreix M (2009) Small-molecule drugs mimicking DNA damage: a new strategy for sensitizing tumors to radiotherapy. Clin Cancer Res 15(4):1308-1316

7. Devun $F$ et al (in press) Assessing chemotherapy efficacy enhancement by inhibition of DSBs repair by Dbait32Hc in colorectal cancers developed in genetically engineered mouse models. J Gastroenterol

8. Reymond MA, Hu B, Garcia A, Reck T, Köckerling F, Hess J, Morel P (2000) Feasibility of therapeutic pneumoperitoneum in a large animal model using a microvaporisator. Surg Endosc 14(1):51-55

9. Solass W, Hetzel A, Nadiradze G, Sagynaliev E, Reymond MA (in press) Description of a novel approach for intraperitoneal drug delivery and the related device. Surg Endosc

10. Piso P (2007) Basisprotokoll für ein multimodales chirurgisches therapiekonzept zur behandlung von patienten mit peritonealcarcinose mittels peritonektomie/multiviszeralresektion und hyperthermer intraperitonealer chemotherapie. Deutsche Gesellschaft für Allgemein-und Viszeralchirurgie, Berlin

11. Guy J, Drabek D, Antoniou M (1995) Delivery of DNA into mammalian cells by receptor-mediated endocytosis and gene therapy. Mol Biotechnol 3(3):237-248 